OPEN ACCESS

Edited by:

Shingo Kajimura,

University of California, San Francisco,

United States

Reviewed by:

Zhipeng (Toby) Tao,

Massachusetts General Hospital and

Harvard Medical School, United States

Yawei Zheng,

Nanjing University of Chinese

Medicine, China

$\mathrm{He} \mathrm{He}$,

Sichuan University, China

*Correspondence:

Amit R. Majithia

amaiithia@ucsd.edu

Specialty section:

This article was submitted to

Obesity,

a section of the journal

Frontiers in Endocrinology

Received: 14 September 2021

Accepted: 23 October 2021

Published: 07 December 2021

Citation:

Du X, DeForest $N$ and Majithia AR (2021) Human Genetics to Identify

Therapeutic Targets for NAFLD:

Challenges and Opportunities.

Front. Endocrinol. 12:777075.

doi: 10.3389/fendo.2021.777075

\section{Human Genetics to Identify Therapeutic Targets for NAFLD: Challenges and Opportunities}

\author{
Xiaomi $\mathrm{Du}^{1,2}$, Natalie DeForest ${ }^{1,3}$ and Amit R. Majithia ${ }^{1 *}$ \\ 1 Division of Endocrinology, Department of Medicine, University of California San Diego, La Jolla, CA, United States, \\ 2 Bioinformatics and Systems Biology Graduate Program, University of California San Diego, La Jolla, CA, United States, \\ ${ }^{3}$ Biomedical Sciences Graduate Program, University of California San Diego, La Jolla, CA, United States
}

Non-alcoholic fatty liver disease (NAFLD) is a continuous progression of pathophysiologic stages that is challenging to diagnose due to its inherent heterogeneity and poor standardization across a wide variety of diagnostic measures. NAFLD is heritable, and several loci have been robustly associated with various stages of disease. In the past few years, larger genetic association studies using new methodology have identified novel genes associated with NAFLD, some of which have shown therapeutic promise. This minireview provides an overview of the heterogeneity in NAFLD phenotypes and diagnostic methods, discusses genetic associations in relation to the specific stages for which they were identified, and offers a perspective on the design of future genetic mapping studies to accelerate therapeutic target identification.

Keywords: NAFLD, NASH, human genetics, gene discovery, GWAS, exomes

\section{INTRODUCTION}

Non-alcoholic fatty liver disease (NAFLD) is the most prevalent liver disease globally, affecting approximately $25 \%$ of the adult population as of 2016 (1), and its incidence continues to increase. NAFLD encompasses simple steatosis (fatty liver; NAFL) and the more severe nonalcoholic steatohepatitis (NASH), which is characterized by fat accumulation, inflammation, and hepatocellular injury. Hepatic fibrosis can develop in NAFLD, which can progress into cirrhosis and hepatocellular carcinoma (HCC) $(2,3)$. As of 2019, NASH was the underlying cause of liver failure in over a third of individuals awaiting liver transplant (4). There are currently no FDA approved treatments for any stage of NAFLD, including NASH (5), highlighting the critical need to identify therapeutic targets.

Given that NAFLD is heritable, with heritability estimates ranging $20 \%-70 \%$ (6), genetic mapping has been undertaken to identify causal genes with potential therapeutic implications. Initial NAFLD studies focused on selected candidate genes, but were limited by small sample size, a high rate of false positive associations due to cryptic population stratification, and reliance on prior knowledge for gene selection (7-9). With the advent of genome-wide association methods that could be applied at population scale, some of these limitations have been overcome, resulting in the unbiased, reproducible genetic discoveries that are detailed below. 
In this mini-review, we focus on the phenotypic complexity of NAFLD, the challenges this poses to executing genetic association studies, and the progress made in identifying new putative targets over the past four years.

\section{NAFLD DEFINITIONS AND DIAGNOSTICS}

NAFLD is a continuum of disease with multiple pathophysiologies and is defined and diagnosed by variable, often incompatible, approaches. In this section, we provide an overview of this heterogeneity in pathogenesis and detection, focusing on how this impacts the interpretation of the genetic associations studies described below. This brief summary does not cover the full breadth of this field, so we refer the reader to other recent reviews for a comprehensive treatment of all diagnostic methods (10), noninvasive diagnostic modalities (11-13), biomarkers (14, 15), and elastography techniques (16).

Clinically, the spectrum of fatty liver disease encapsulated in NAFLD is defined in the absence of excess alcohol intake (5). The distinction between NAFL and NASH is most commonly differentiated by the absence (NAFL) or presence (NASH) of hepatocyte ballooning (17). Some studies further delineate phases between NAFL and NASH $(18,19)$, and between NASH and cirrhosis (20), highlighting the continuum of pathophysiology. For simplicity, this mini-review will anchor on three stages of NAFLD - NAFL, NASH, and cirrhosis as depicted in Figure 1.

\section{Histology Is the Gold Standard}

Liver biopsies are the gold standard for NAFLD diagnosis, and the FDA requires evidence of histologic improvement for NAFLD treatments in late stage clinical trials for consideration of approval (47). In clinical research, biopsies are commonly graded by the NAFLD Activity Score (NAS), which quantifies NAFLD severity based on steatosis (0-3), lobular inflammation (0-3), hepatocyte ballooning (0-2), and fibrosis (0-4) (48) (Figure 1). NAS was not designed to be a diagnostic tool, so defining NASH by a cut off threshold of NAS $\geq 5$ can result in inaccurate diagnoses $(49,50)$. There are also different scoring criteria, including the Brunt score (51) and the steatosis-activityfibrosis (SAF) score (52), that can be used to grade biopsies, and this lack of a single standard leads to difficulties in comparisons between studies. This is further exacerbated by sampling variability due to histologic heterogeneity (53) and subjectivity in interpretation for liver biopsies (54-56). This gold standard based on tissue sampling also limits the investigation of NAFLD at scale for large cohort studies, and biopsies are often refused by patients in clinical practice (15). Thus, there has been a strong emphasis by clinicians and researchers on the development of alternative, noninvasive diagnostic techniques.

\section{Noninvasive Methods of Diagnosis}

There are many imaging methods to detect hepatic steatosis, including computed tomography (CT), magnetic resonance imaging-proton density fat fraction (MRI-PDFF), and proton magnetic resonance spectroscopy $\left({ }^{1} \mathrm{H}-\mathrm{MRS}\right)$ (Figure 1). CT can quantitatively measure liver fat content, but it has poor sensitivity for mild steatosis (57), and it exposes patients to ionizing radiation $(58,59)$. MRI-PDFF and ${ }^{1} \mathrm{H}-\mathrm{MRS}$ both measure steatosis with high accuracy relative to histologic references, even at low amounts of hepatic fat, so they are the recommended imaging modalities for liver fat (60-62).

Although these imaging methods can accurately measure steatosis, they are poorly suited to detect the features differentiating NASH from NAFL, i.e., ballooning, inflammation, and fibrosis (63). A recently published protocol for multiparametric magnetic resonance (MR) has bridged that gap. MR derived iron-corrected T1 (cT1) is a novel noninvasive method to assess fibrosis (64), and it correlates with all the histological features of NASH $(65,66)$.

Liver enzyme levels have also been correlated with NASH and fibrosis (67). The classic indications of liver inflammation are aspartate aminotransferase (AST), alanine aminotransferase (ALT), gamma-glutamyltransferase (GGT), and alkaline phosphatase (ALP), along with the AST/ALT ratio $(68,69)$ (Figure 1). Elevated enzyme levels are insufficient to provide a confident NAFLD diagnosis, however, because ALT values are normal in up to $25 \%$ of NAFLD patients $(70,71)$.

Altogether, no single noninvasive method has replaced histology yet for detection of all the phenotypes characteristic of NAFLD. Nevertheless, studies have effectively employed combinations of these alternative modalities to measure the full spectrum of NAFLD features.

\section{GENETIC APPROACHES FOR NAFLD THERAPEUTIC TARGET IDENTIFICATION}

Epidemiological, familial aggregation, and twin studies over the past two decades have demonstrated a heritable component to NAFLD (72), strongly suggesting that genetic mapping approaches could be productively deployed to identify genes with therapeutic potential. As mentioned earlier, initial genetic investigations into NAFLD utilized candidate gene approaches, but the development of next-generation sequencing (NGS) and high-throughput genotyping arrays enabled more robust, unbiased methods of genetic mapping studies including genome-wide association studies (GWAS) and exome-wide association studies (EWAS) $(73,74)$. GWAS has successfully identified loci that are associated with risk for many complex diseases and traits using common variants ascertained from genotyping (75), whereas EWAS examines variants predominantly in the exonic (i.e. protein-coding) regions of the genome (76). With the decreasing cost of NGS, current studies can detect exonic variants through whole-exome sequencing (WES) (77). Recent expert reviews have summarized variants identified from NAFLD genetic association studies $(6,78,79)$. Here, we build upon these publications by reviewing the literature from the past four years, highlighting the consequence of NAFLD phenotypic heterogeneity on genetic discovery, and quantifying the limits of current association studies to identify new genetic signals. 


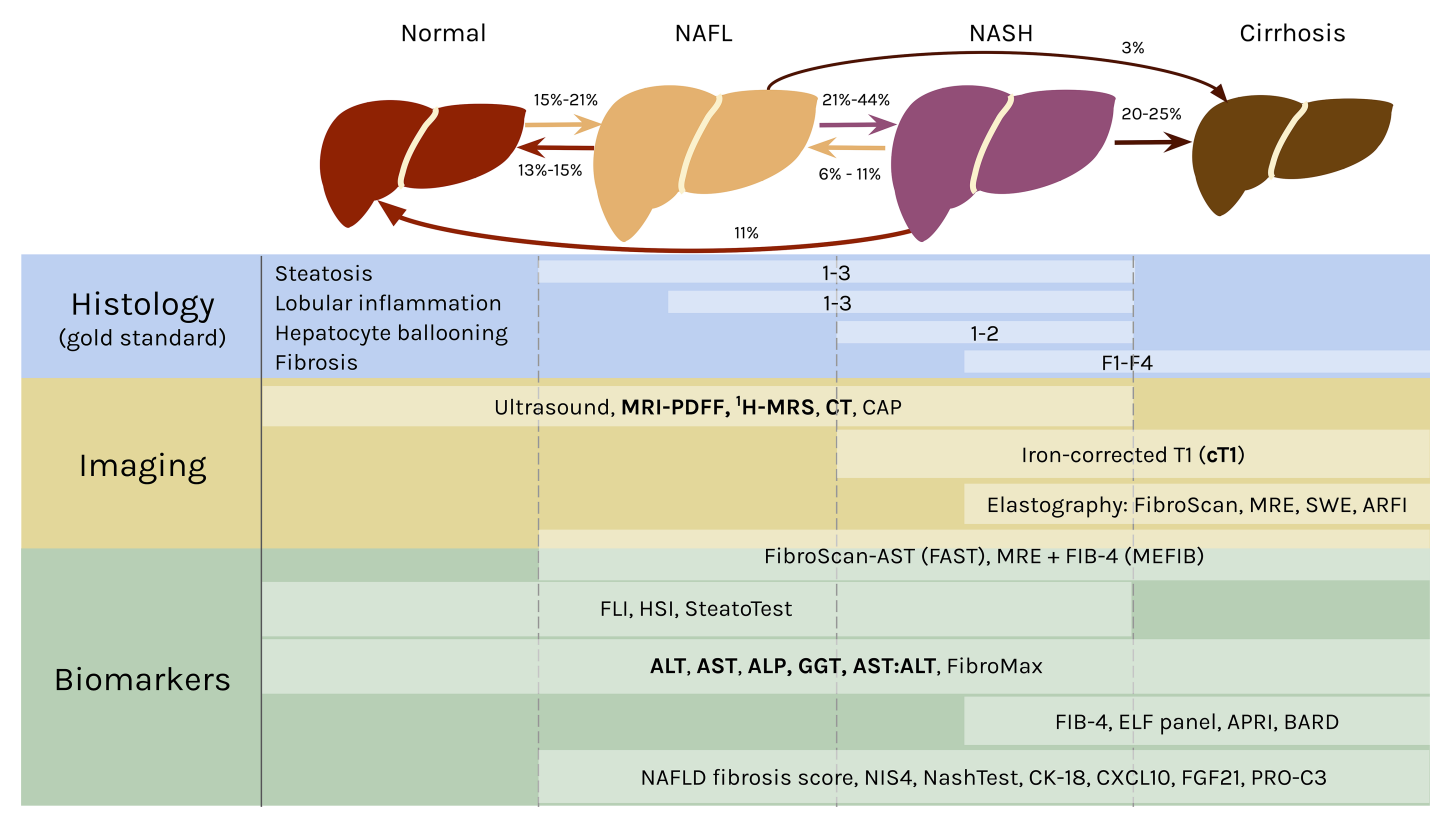

FIGURE 1 | The spectrum of NAFLD and stage specific clinical measures. In NAFL, at least $5 \%$ of the hepatocytes have fat accumulation in the form of large lipid droplets in the cells that displace the nuclei (21) or many small lipid droplets (22). NAFL can also include inflammation. The transition to NASH occurs with hepatocellular injury in the form of ballooning and further inflammation. Fibrosis can develop in NASH and advance into cirrhosis, in which the liver shrinks and hardens. NAFL and NASH are reversible, as indicated by the rates regressing in severity. Both can also progress into cirrhosis. The rates of transition between each stage are broad ranges because they originate from studies with varying cohort sizes, time frames, treatments, and other variables (18, 19, 23-26). Histology is the gold standard for diagnosing NAFLD and classifying the stage of disease. The components of NAS are listed above, with the area to the left of the white bars indicating scores of 0 for each feature. A selection of noninvasive diagnostic methods are shown below, with white boxes representing their range in effectively diagnosing different stages of NAFLD [ultrasound (27); FAST (28); MEFIB (29); SteatoTest (30); FibroMax (31); BARD (32); NAFLD fibrosis score (33); NIS4 (34); NASHTest (35)]. The lists are not comprehensive, and the modalities mentioned in the mini-review are emphasized in bold. CAP, controlled attenuation parameter; obtained from FibroScan $(36,37)$. MRE, magnetic resonance elastography (38). SWE, shear-wave elastography (38). ARFI, acoustic radiation force impulse (37). FIB-4, fibrosis-4 (39). FLI, fatty liver index (40). HSI, hepatic steatosis index (41). ELF, Enhanced Liver Fibrosis (42). APRI, AST-to-platelet ratio index (43). CK-18, cytokeratin 18 (44). CXCL10, C-X-C motif chemokine ligand 10 (45). FGF21, fibroblast growth factor 21 (44). PRO-C3, plasma collagen type III (46).

\section{Genetic Associations Discovered in the Past Four Years}

We focus our attention on novel loci discovered in NAFLD related GWAS and EWAS from the past four years.

Abul-Husn et al. performed an EWAS for ALT and AST levels using WES $(n=46,544)$ and validated their associations in two additional cohorts $(\mathrm{n}=9,883)$ and liver biopsy samples $(n=2,391)(80)$. They found that a loss of function, proteintruncating variant in HSD17B13 (rs72613567:TA) was associated with decreased levels of ALT and AST and lower rates of NASH, as determined by the presence of any inflammation or hepatocyte ballooning in liver histology. At the same time, this variant was not associated with NAFL (80), providing evidence that HSD17B13 may be involved in more clinically advanced stages of NAFLD.

Namjou at el. used a natural language processing (NLP) algorithm to identify NAFLD cases for a GWAS in pediatric and adult cohorts (1,106 cases and 8,571 controls) (81). They replicated associations between NAFLD and variants in the PNPLA3-SAMM50-PARVB locus (including rs738409). Namjou et al. subsequently performed quantitative case-only association studies for NAS, fibrosis, AST and ALT, finding that
IL17RA was associated with NAS, and ZFP90-CDH1 was associated with fibrosis.

Anstee et al. conducted the largest GWAS to date for NAFLD ascertained by histology (1,483 cases and 17,781 controls) and identified two new associations (82). An intronic variant near the LEPR gene was associated with NASH at genome-wide significance, and a missense variant in PYGO1 encoding p.P299H (rs11858624) was associated with protection from steatosis at close to genome-wide significance (82).

Parisinos et al. performed a GWAS for liver inflammation and fibrosis using cT1 values $(\mathrm{n}=14,440)$ and studied the associations between significant variants and liver biomarkers $(\mathrm{n}=378,821)$. Novel variants in SLC30A10 and SLC39A8 had genome-wide significant associations with $\mathrm{CT} 1$ and elevated levels of ALT and AST. In a separate GWAS performed on the same cohort $(n=14,440)$, four variants were associated with steatosis measured by MRI-PDFF, including APOE rs429358, a missense variant that encodes p.C112R. Parisinos et al. further studied the associations between $\mathrm{cT} 1$ values and variants identified by a cirrhosis GWAS, which found a missense variant in MARC1 encoding p.A165T (rs2642438) that protects against cirrhosis (83). This analysis revealed that variants in 
MARC1 and HSD17B13 were associated with both cirrhosis and cT1 values (84).

A recent study of protein-coding variants ascertained by genotyping arrays investigated genetic associations for ALT levels $(n=425,671)(85)$. The authors found 190 genetic variants associated with ALT, replicated their findings in three public GWAS databases, and associated the variants with liver fat as measured by MRI-PDFF $(n=8,930)$ to validate significant variants. These variants, including single nucleotide polymorphisms (SNPs) in MARC1, APOE, and GPAM, were all additionally associated with chronic liver disease and cirrhosis. Jamialahmadi et al. further validated these genetic associations with liver biopsies $(n=2,621)$. The missense variant in GPAM (rs2792751) encoding p.V43I was found to be significantly associated with severity of liver steatosis, while APOE rs429358 confers protection for liver steatosis. The association between APOE and NAFLD was also found in an exome-wide association meta-analysis of CT-measured liver steatosis across eight multi-ethnic population-based cohorts $(n=16,492)(86)$.

Pazoki et al. performed GWAS on serum levels of ALT, ALP, and GGT $(n=437,438)$ and replicated their results in three additional cohorts $(n=315,572)(87)$. These enzymatic indicators of inflammation and liver disease were associated with 517 SNPs, including variants in SERPINA1, APOE, GPAM, MARC1, and LEPR. The number of variants associated with any combination of ALT, ALP, and GGT is likely greater than the number found by studies that used imaging or histology to assess NAFLD because serum levels are not specific to NAFLD and are reflective of many processes in the body, including cardiovascular disease (87).

Liu et al. applied deep learning to MRI scans to quantify volume, fat, and iron in many organs, including the liver $(n=38,881)$, and performed GWAS on their results $(n=32,858$ for liver fat). Variants near $P P P 1 R 3 B$ and in GCKR were associated with liver volume, which was strongly correlated with liver function (88). Liu et al. also identified eight variants associated with liver fat, including TRIB1 rs112875651, MARC1 rs2642438, GPAM rs11446981, and a region in MTTP.

\section{Genetic Associations in the Context of NAFLD Phenotypic and Diagnostic Heterogeneity}

Multiple GWAS and EWAS have been conducted to find genetic associations with specific features of NAFLD, such as hepatic steatosis, fibrosis, and liver inflammation, as well as the full spectrum of disease. Some variants have been associated with the full NAFLD spectrum, while others are only correlated with certain phenotypes. The specific NAFLD phenotypes and measurements that these genes have been associated with through GWAS and/or EWAS are summarized in Figure 2.

A missense SNP in PNPLA3 (rs738409) encoding p.I148M is the most robustly associated genetic variant with the full spectrum of NAFLD (78). The landmark PNPLA3 study measured hepatic fat content by ${ }^{1} \mathrm{H}$-MRS and examined inflammation through serum levels of ALT to suggest that

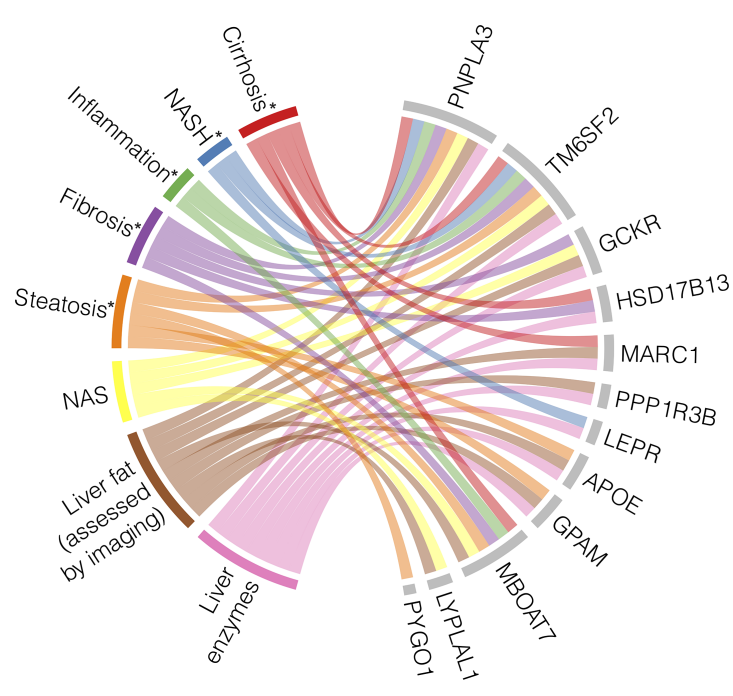

FIGURE 2 | Heterogeneity and pleiotropy of genes associated with NAFLD phenotypes. Loci with their corresponding genes which have been shown to be associated with NAFLD through genome- and/or exome-wide association studies are displayed on the right side of the chord diagram, indicated by grey bars. On the left side of the diagram are the NAFLD states with their associated measurements, each represented by a different color. Each ribbon represents a significant association identified between each gene and the described state. * indicates histologically defined state $(56,80,82,84-87$, 89-96).

rs738409 could increase risk of NASH, but the study itself did not focus on histologic NASH or cirrhosis (97). Since then, many GWAS and EWAS have identified a relationship between PNPLA3 and steatosis measured by other imaging methods (86), histologically defined steatosis, hepatocyte ballooning, lobular inflammation, fibrosis, and cirrhosis $(82,89)$ and cT1 defined NASH (84).

Similarly, the missense variant rs58542926 encoding p.E167K in TM6SF2 was initially associated with hepatic fat measured by ${ }^{1} \mathrm{H}$-MRS and liver enzyme levels (90), and it has since been found to associate with the full range of NAFLD phenotypes. TM6SF2 rs58542926 has been robustly associated with steatosis assessed by CT in independent studies $(84,86)$. The initial AST, ALT, and ALP associations were replicated by Parisinos et al. and further supported by associations between the variant and cT1 values (84) and histologically ascertained NAS and SAF scores (91), indicating that TM6SF2 rs58542926 is implicated in NASH in addition to NAFL. TM6SF2 was also associated with histologically graded cirrhosis (84).

Several other loci have been pleiotropically associated with multiple NAFLD stages. GCKR rs1260326 is associated with hepatic steatosis, as assessed by both imaging and histology $(84,86,92,93)$, inflammation measured by serum enzyme levels $(87,92)$, histological assessments of NAFLD graded by NAS (94), histological fibrosis (82), and overall liver function (88). MBOAT7 has similarly been examined across the entire spectrum of NAFLD, ranging from liver fat accumulation to 
cirrhosis $(85,95,98)$, but intriguingly, it was found to be independently associated with fibrosis development in particular, suggesting a unique molecular mechanism (56). Finally, MARC1 has been associated with steatosis $(85,88)$, inflammation/NASH $(84,85,87)$, and cirrhosis $(83,84)$.

Conversely, some genetic associations have been identified for only specific NAFLD stages and diagnostic modalities. As described above, $P Y G O 1$ has only been associated with histologically identified steatosis (82). GPAM, PPP1R3B, and $A P O E$ have associations with steatosis $(84-86,88,93,94)$ and serum enzyme levels $(85,87,92)$, but these loci have not been associated with histological features of NASH or cirrhosis. On the other hand, LEPR is only associated with ALT levels and histologically defined NASH $(82,87)$, and HSD17B13 is associated with NASH and cirrhosis $(80,84)$.

There are some variants that have only been identified in a single study so far, introducing uncertainty in their relationship with NAFLD. For example, variant rs12137855 mapped to LYPLAL1 has been associated with liver fat and histologic NAFLD, as quantified by NAS (94), but this SNP has not been replicated in this past decade by other association studies. A possible explanation for this lack of reproducibility is the combination of the small effect size of the LYPLAL1 variant and the current limits in statistical power.

\section{CURRENT STUDY DESIGN LIMITATIONS TO DISCOVERING NAFLD ASSOCIATED VARIANTS}

The variants identified through GWAS and EWAS are susceptible to the study design choices. The sample size, diversity within the cohort, and specificity of the associated trait, along with many other confounders, can all affect the results (99). A major cofounder is that sample size in current NAFLD studies is highly correlated to the measurement modality. On the spectrum of sample numbers, liver enzyme levels, which are commonly available as part of routine blood testing, are on the high end, and liver biopsies, which require a clinical indication and are difficult to perform in large numbers, are on the low end (63). Because statistical power to detect significant associations is directly dependent on sample size (100), studies using biopsies are often underpowered, while studies using serum concentrations are better powered but less informative for NAFLD stages.

In Figure 3 (left panel), we illustrate the statistical power of the largest liver biopsy GWAS to date (82), which included 1,483 NAFLD biopsied cases and 17,781 controls. Given its size, this study would be predicted to successfully replicate previously characterized loci, including PNPLA3, TM6SF2, HSD17B13, and $G C K R$, based on their respective frequencies in the population and effect sizes on NAFLD risk. These associations are indeed found with genome-wide significance (82). Furthermore, a GWAS of this size would be predicted to not detect MARC1 and LYPLAL1 with genome-wide significance, as those variants have smaller effect sizes. Again, this is reflected in the results: although the variant in MARC1 was associated with NAFLD with $p<6 \times 10^{-6}$, the association did not meet the genome-wide significance threshold (82).

Future studies with increased sample sizes of NAFLD individuals and balanced case/control designed studies may reveal novel genetic associations which studies are currently underpowered to detect and provide additional support for existing associations (Figure 3, right panel). With the generation of larger NAFLD case/control cohorts and increased application of WES, more rare variants with large biological effects can be identified, which would facilitate therapeutic targeting. Rare, loss-of-function variants that confer protection from disease in particular have shown promise as therapeutic targets, as exemplified by the successful development of PCSK9 inhibitors to treat atherogenic cardiovascular disease $(101,102)$.

\section{CURRENT PERSPECTIVE ON NAFLD ASSOCIATION STUDIES}

Identifying causal genes is a major challenge to translating genetic association signals into biological and potentially therapeutic knowledge. The majority of variants identified from GWAS are located in non-coding genomic sequences distant from protein-coding genes (99). For example, a variant on chromosome 8 that lies in the intergenic region between IDO2 and TC1 was associated with NAFLD, but it is unclear which gene is driving the phenotype (82). Additionally, while it is standard practice to designate the nearest gene to a variant as the causal gene, this may not always be true. This caveat was showcased by the variant rs2075650 residing in an intron of TOMM40, which was found to be associated with steatosis. While most proximal to the TOMM40 coding sequences, conditional analysis showed that this variant association was driven by linkage disequilibrium with the previously identified APOE rs429358 (86). In contrast to GWAS, EWAS analyses almost entirely use variants in the exonic regions of the genome, which can make causal gene identification more straightforward as the variants likely alter the sequence of the encoded protein. However, to date, the findings of NAFLD EWAS are still limited. So far, only one study specific to NAFLD has harnessed rare protein-coding genetic variants from WES rather than genomeor exome- arrays (103), but additional large-scale studies of rare variants and their effects on NAFLD are beginning to emerge and find new signals such as MAST3 and IFI30 (104, 105).

As mentioned earlier, increasing sample size to power robust discovery is a current challenge in NAFLD gene discovery due in large part to inherent limitations in the scalability of liver biopsies. Some studies have employed creative methods to increase sample sizes for their NAFLD genetic studies with some indications of success. These techniques include NLP algorithms (81), machine learning applied to liver imaging (88, 104), and a multi-step approach of first identifying genetic signals with a widely available biomarker, such as liver enzyme levels, in population cohorts, and then examining only these 


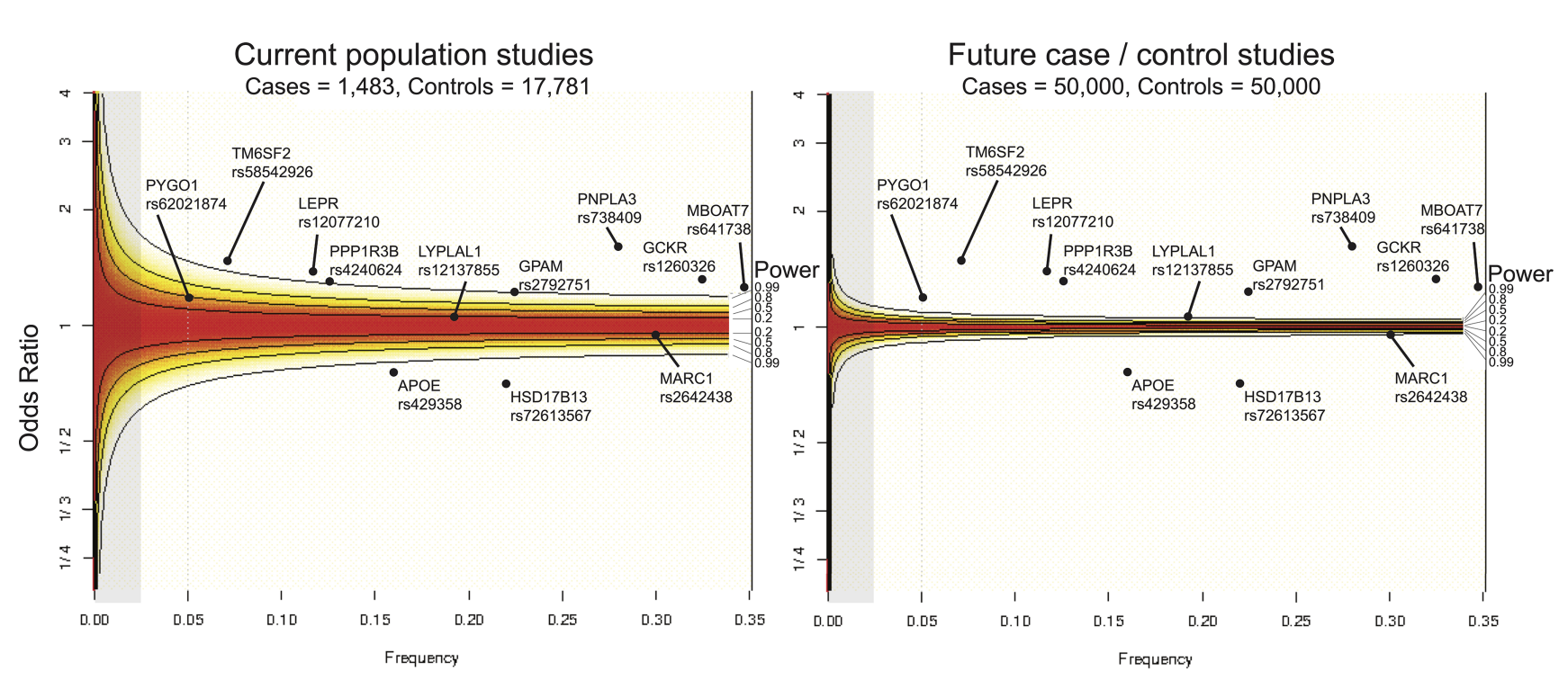

FIGURE 3 | Current state of statistical power to detect genetic associations with NAFLD. Statistical power across odds ratios per allele frequencies computed with a type 1 error rate set at $\alpha=0.05$ shown for current sample size of NAFLD population studies (left) and for an idealized future NAFLD case/control study (right). Power for frequencies and odds ratios regions are shaded from red to white, in which red indicates regions where statistical power is not sufficient to detect an association, and white areas are within the detection limit. Gray areas highlight the section of the power curve for associating rare genetic variants (MAF < 0.025). Associated loci are overlaid according to their effect size and minor allele frequencies. For example, PNPLA3 rs62021874, which has been robusted associated with NAFLD across several studies, is well within the detectable region given its minor allele frequency of 0.28 and odds ratio of 1.8 , whereas PYGO1 (frequency $=0.05$, odds ratio $=1.3$ ), which has been associated in a single study, is nominally powered to detect an association given the current sample size of NAFLD population studies $(56,82,85,93)$.

identified signals with independent histological cohorts $(80,94)$ to decrease the multiple hypothesis correction burden. For example, Abul-Husn et al. first conducted a GWAS in almost 47,000 individuals to identify variants significantly associated with either ALT or AST levels (80). 13 of these variants were next replicated in an additional cohort $(n=12,527)$, and then these top variants were identified within exome sequences $(n=1,857$ NAFLD cases and 29,928 controls) and tested for association with chronic liver disease. This reduced the statistical threshold for significance without increasing the false positive rate. From this targeted exome association analysis, the protein-truncating variant in HSD17B13 (rs72613567) was found to confer lower odds across all categories of liver disease and provide protection against liver fibrosis in an allele dose-dependent manner. This discovery then led to the development of ARO-HSD, a RNAi therapeutic that selectively targets HSD17B13 mRNA in hepatocytes, which has demonstrated improvements in NASH outcomes, as assessed by ALT, AST, and MRI-PDFF, in a Phase $1 / 2$ clinical trial $(106,107)$.

In the serendipitous case of $H S D 17 B 13$, the consequence of the top identified variant was protein-truncating and thus could be predicted with high confidence to confer loss of function in HSD17B13 without additional functional characterization. In order to provide analogous interpretations to genetic variants that do not have such clear cut functional effects without performing validation experiments, NAFLD genetic studies have utilized computational prediction tools (108), ClinVar reported pathogenicity predictions, and allele frequency cut- offs (109), to narrow the search space to actionable variants, but with limited success in the absence of mechanistic investigation. In summary, despite current limitations, genetic discoveries for NAFLD have demonstrated promise in therapeutic target identification. Future genetic investigations with increased sample size and focusing on different stages of NAFLD are likely to reveal new genes with therapeutic potential.

\section{FUTURE DIRECTIONS}

Efforts are underway to improve standardization in classification and diagnosis of NAFLD to enable translational research that can identify putative drug targets. In 2020, an expert consensus panel proposed a new set of diagnostic criteria for NAFLD (110) and renamed it metabolic associated fatty liver disease (MAFLD). MAFLD is diagnosed by the presence of hepatic steatosis (ascertained by imaging, biomarker panel or histology) and either type 2 diabetes (T2D) or overweight/obesity, or two of the following metabolic risk factors: waist circumference, blood pressure, serum triglycerides, low serum HDL, prediabetes, insulin resistance, and plasma high-sensitivity $\mathrm{C}$-reactive protein level (111). Recent publications indicate that the MAFLD criteria performs better than the NAFLD definition at identifying patients with more severe presentations of disease (112-114), but the new terminology is still heavily debated (115). To date, one genetic association study has been performed using 
the MAFLD definition and recapitulated the known genetic associations with PNPLA3 rs738409 and TM6SF2 rs8542926 (116). This is promising as MAFLD diagnosis does not require biopsies and can be diagnosed from readily available clinical measurements. Nevertheless, further validation is required, especially for the SNPs associated with hepatic injury and fibrosis, which are not explicitly included in the MAFLD definition.

Other than the RNAi targeting HSD17B13 mRNA mentioned above, most therapeutics currently in clinical trials do not target genes identified from GWAS (63). One possible direction to identify novel, actionable targets for NAFLD from gene or exome wide associations would entail a combination of imaging and biomarkers for NAFLD diagnostic staging that could be broadly applied to hundreds of thousands of individuals in biobanks, as demonstrated by recent publications $(84,85)$. A specific pathophysiology of NAFLD, such as NASH defined by MRI-PDFF and cT1, should be selected to ensure that there is a sufficiently large cohort of cases for a well-powered study. Association analysis could then be performed to identify rare variants with large effect sizes associated with this classification of NASH. The variants could be further investigated by functional validation in molecular assays to find the causal genes, which would then be the targets of drug development.

\section{REFERENCES}

1. Younossi ZM, Koenig AB, Abdelatif D, Fazel Y, Henry L, Wymer M. Global Epidemiology of Nonalcoholic Fatty Liver Disease-Meta-Analytic Assessment of Prevalence, Incidence, and Outcomes. Hepatology (2016) 64:73-84. doi: 10.1002/hep.28431

2. Anstee QM, Targher G, Day CP. Progression of NAFLD to Diabetes Mellitus, Cardiovascular Disease or Cirrhosis. Nat Rev Gastroenterol Hepatol (2013) 10:330-44. doi: 10.1038/nrgastro.2013.41

3. Huang DQ, El-Serag HB, Loomba R. Global Epidemiology of NAFLDRelated HCC: Trends, Predictions, Risk Factors and Prevention. Nat Rev Gastroenterol Hepatol (2021) 18:223-38. doi: 10.1038/s41575-020-00381-6

4. Wong RJ, Singal AK. Trends in Liver Disease Etiology Among Adults Awaiting Liver Transplantation in the United States, 2014-2019. JAMA Netw Open (2020) 3:e1920294. doi: 10.1001/jamanetworkopen. 2019.20294

5. Chalasani N, Younossi Z, Lavine JE, Charlton M, Cusi K, Rinella M, et al. The Diagnosis and Management of Nonalcoholic Fatty Liver Disease: Practice Guidance From the American Association for the Study of Liver Diseases. Hepatology (2018) 67:328-57. doi: 10.1002/hep.29367

6. Eslam M, George J. Genetic Contributions to NAFLD: Leveraging Shared Genetics to Uncover Systems Biology. Nat Rev Gastroenterol Hepatol (2020) 17:40-52. doi: 10.1038/s41575-019-0212-0

7. Sillanpää MJ. Overview of Techniques to Account for Confounding Due to Population Stratification and Cryptic Relatedness in Genomic Data Association Analyses. Heredity (2011) 106:511-9. doi: 10.1038/hdy.2010.91

8. Sookoian S, Pirola CJ. Genetic Predisposition in Nonalcoholic Fatty Liver Disease. Clin Mol Hepatol (2017) 23:1-12. doi: 10.3350/cmh.2016.0109

9. Cardon LR, Bell JI. Association Study Designs for Complex Diseases. Nat Rev Genet (2001) 2:91-9. doi: 10.1038/35052543

10. Younossi ZM, Loomba R, Anstee QM, Rinella ME, Bugianesi E, Marchesini G, et al. Diagnostic Modalities for Nonalcoholic Fatty Liver Disease, Nonalcoholic Steatohepatitis, and Associated Fibrosis. Hepatology (2018) 68:349-60. doi: 10.1002/hep.29721

11. Castera L, Friedrich-Rust M, Loomba R. Noninvasive Assessment of Liver Disease in Patients With Nonalcoholic Fatty Liver Disease. Gastroenterology (2019) 156:1264-81.e4. doi: 10.1053/j.gastro.2018.12.036

\section{AUTHOR CONTRIBUTIONS}

$\mathrm{XD}$ and $\mathrm{AM}$ conceived the manuscript outline and figure concepts. XD and ND authored sections of the manuscript and created figures. $\mathrm{XD}, \mathrm{ND}$, and $\mathrm{AM}$ were involved in critical manuscript revision. All authors contributed to the article and approved the submitted version.

\section{FUNDING}

This work was supported by grants from the National Institute of Diabetes and Digestive and Kidney Diseases (1R03DK113328-01 and 1R01DK123422-01 to ARM), a UCSD/UCLA Pilot and Feasibility grant (P30 DK063491 to ARM), and a Ruth L. Kirschstein Institutional National Research Service Award T32 GM008666 from the National Institute of General Medical Sciences (to ND).

\section{ACKNOWLEDGMENTS}

We thank Jason Flannick for helpful discussions in visualizing statistical power for existing and hypothetical genetic association studies as shown in Figure 3.

12. Zhou J-H, Cai J-J, She Z-G, Li H-L. Noninvasive Evaluation of Nonalcoholic Fatty Liver Disease: Current Evidence and Practice. World J Gastroenterol (2019) 25:1307-26. doi: 10.3748/wjg.v25.i11.1307

13. Piazzolla VA, Mangia A. Noninvasive Diagnosis of NAFLD and NASH. Cells (2020) 9:1005. doi: 10.3390/cells9041005

14. Wong VW-S, Adams LA, de Lédinghen V, Wong GL-H, Sookoian S. Noninvasive Biomarkers in NAFLD and NASH - Current Progress and Future Promise. Nat Rev Gastroenterol Hepatol (2018) 15:461-78. doi: 10.1038/s41575-018-0014-9

15. Tapper EB, Loomba R. Noninvasive Imaging Biomarker Assessment of Liver Fibrosis by Elastography in NAFLD. Nat Rev Gastroenterol Hepatol (2018) 15:274-82. doi: 10.1038/nrgastro.2018.10

16. Honda Y, Yoneda M, Imajo K, Nakajima A. Elastography Techniques for the Assessment of Liver Fibrosis in Non-Alcoholic Fatty Liver Disease. Int J Mol Sci (2020) 21:4039. doi: 10.3390/ijms21114039

17. Sanyal AJ, Brunt EM, Kleiner DE, Kowdley KV, Chalasani N, Lavine JE, et al. Endpoints and Clinical Trial Design for Nonalcoholic Steatohepatitis. Hepatology (2011) 54:344-53. doi: 10.1002/hep.24376

18. Wong VW-S, Wong GL-H, Choi PC-L, Chan AW-H, Li MK-P, Chan H-Y, et al. Disease Progression of Non-Alcoholic Fatty Liver Disease: A Prospective Study With Paired Liver Biopsies at 3 Years. Gut (2010) 59:969-74. doi: 10.1136/gut.2009.205088

19. Kleiner DE, Brunt EM, Wilson LA, Behling C, Guy C, Contos M, et al. Association of Histologic Disease Activity With Progression of Nonalcoholic Fatty Liver Disease. JAMA Netw Open (2019) 2:e1912565. doi: 10.1001/ jamanetworkopen.2019.12565

20. Anstee QM, Reeves HL, Kotsiliti E, Govaere O, Heikenwalder M. From NASH to HCC: Current Concepts and Future Challenges. Nat Rev Gastroenterol Hepatol (2019) 16:411-28. doi: 10.1038/s41575-019-0145-7

21. Anstee QM, Day CP. 26 - Epidemiology, Natural History, and Evaluation of Nonalcoholic Fatty Liver Disease. In: Sanyal AJ, Boyer TD, Lindor KD, Terrault NA, editors. Zakim and Boyer's Hepatology (Seventh Edition). Philadelphia: Elsevier (2018). p. 391-405.e3. doi: 10.1016/B978-0-323-37591-7.00026-4

22. Tandra S, Yeh MM, Brunt EM, Vuppalanchi R, Cummings OW, ÜnalpArida A, et al. Presence and Significance of Microvesicular Steatosis in Nonalcoholic Fatty Liver Disease. J Hepatol (2011) 55:654-9. doi: 10.1016/ j.jhep.2010.11.021 
23. McPherson S, Hardy T, Henderson E, Burt AD, Day CP, Anstee QM. Evidence of NAFLD Progression From Steatosis to Fibrosing-Steatohepatitis Using Paired Biopsies: Implications for Prognosis and Clinical Management. J Hepatol (2015) 62:1148-55. doi: 10.1016/j.jhep.2014.11.034

24. Diehl AM, Day C. Cause, Pathogenesis, and Treatment of Nonalcoholic Steatohepatitis. N Engl J Med (2017) 377:2063-72. doi: 10.1056/ NEJMra1503519

25. Heyens LJM, Busschots D, Koek GH, Robaeys G, Francque S. Liver Fibrosis in Non-Alcoholic Fatty Liver Disease: From Liver Biopsy to Non-Invasive Biomarkers in Diagnosis and Treatment. Front Med (2021) 8:615978. doi: 10.3389/fmed.2021.615978

26. Drew L. Fighting the Fatty Liver. Nature (2017) 550:S102-3. doi: 10.1038/ 550 S102a

27. Hernaez R, Lazo M, Bonekamp S, Kamel I, Brancati FL, Guallar E, et al. Diagnostic Accuracy and Reliability of Ultrasonography for the Detection of Fatty Liver: A Meta-Analysis. Hepatology (2011) 54:1082-90. doi: 10.1002/ hep. 24452

28. Newsome PN, Sasso M, Deeks JJ, Paredes A, Boursier J, Chan W-K, et al. Fibroscan-AST (FAST) Score for the Non-Invasive Identification of Patients With Non-Alcoholic Steatohepatitis With Significant Activity and Fibrosis: A Prospective Derivation and Global Validation Study. Lancet Gastroenterol Hepatol (2020) 5:362-73. doi: 10.1016/S2468-1253(19)30383-8

29. Tamaki N, Imajo K, Sharpton S, Jung J, Kawamura N, Yoneda M, et al. MRE Plus FIB-4 (MEFIB) Versus FAST in Detection of Candidates for Pharmacological Treatment of NASH-Related Fibrosis. Hepatology (2021). doi: 10.1002/hep.32145

30. Poynard T, Ratziu V, Naveau S, Thabut D, Charlotte F, Messous D, et al. The Diagnostic Value of Biomarkers (Steatotest) for the Prediction of Liver Steatosis. Comp Hepatol (2005) 4:10. doi: 10.1186/1476-5926-4-10

31. Morra R, Munteanu M, Imbert-Bismut F, Messous D, Ratziu V, Poynard T. Fibromax: Towards a New Universal Biomarker of Liver Disease? Expert Rev Mol Diagn (2007) 7:481-90. doi: 10.1586/14737159.7.5.481

32. Harrison SA, Oliver D, Arnold HL, Gogia S, Neuschwander-Tetri BA. Development and Validation of a Simple NAFLD Clinical Scoring System for Identifying Patients Without Advanced Disease. Gut (2008) 57:1441-7. doi: 10.1136/gut.2007.146019

33. Angulo P, Hui JM, Marchesini G, Bugianesi E, George J, Farrell GC, et al. The NAFLD Fibrosis Score: A Noninvasive System That Identifies Liver Fibrosis in Patients With NAFLD. Hepatology (2007) 45:846-54. doi: 10.1002/hep.21496

34. Harrison SA, Ratziu V, Boursier J, Francque S, Bedossa P, Majd Z, et al. A Blood-Based Biomarker Panel (NIS4) for Non-Invasive Diagnosis of NonAlcoholic Steatohepatitis and Liver Fibrosis: A Prospective Derivation and Global Validation Study. Lancet Gastroenterol Hepatol (2020) 5:970-85. doi: 10.1016/S2468-1253(20)30252-1

35. Poynard T, Ratziu V, Charlotte F, Messous D, Munteanu M, Imbert-Bismut F, et al. Diagnostic Value of Biochemical Markers (Nashtest) for the Prediction of Non Alcoholo Steato Hepatitis in Patients With NonAlcoholic Fatty Liver Disease. BMC Gastroenterol (2006) 6:34. doi: 10.1186/1471-230X-6-34

36. de Lédinghen V, Wong GL-H, Vergniol J, Chan HL-Y, Hiriart J-B, Chan AW-H, et al. Controlled Attenuation Parameter for the Diagnosis of Steatosis in Non-Alcoholic Fatty Liver Disease. J Gastroenterol Hepatol (2016) 31:848-55. doi: 10.1111/jgh.13219

37. Cassinotto C, Boursier J, de Lédinghen V, Lebigot J, Lapuyade B, Cales P, et al. Liver Stiffness in Nonalcoholic Fatty Liver Disease: A Comparison of Supersonic Shear Imaging, Fibroscan, and ARFI With Liver Biopsy. Hepatology (2016) 63:1817-27. doi: 10.1002/hep.28394

38. Furlan A, Tublin ME, Yu L, Chopra KB, Lippello A, Behari J. Comparison of 2D Shear Wave Elastography, Transient Elastography, and MR Elastography for the Diagnosis of Fibrosis in Patients With Nonalcoholic Fatty Liver Disease. AJR Am J Roentgenol (2020) 214:W20-6. doi: 10.2214/AJR.19.21267

39. Shah AG, Lydecker A, Murray K, Tetri BN, Contos MJ, Sanyal AJ, et al. Comparison of Noninvasive Markers of Fibrosis in Patients With Nonalcoholic Fatty Liver Disease. Clin Gastroenterol Hepatol (2009) 7:1104-12. doi: 10.1016/j.cgh.2009.05.033

40. Bedogni G, Bellentani S, Miglioli L, Masutti F, Passalacqua M, Castiglione A, et al. The Fatty Liver Index: A Simple and Accurate Predictor of Hepatic
Steatosis in the General Population. BMC Gastroenterol (2006) 6:33. doi: 10.1186/1471-230X-6-33

41. Lee J-H, Kim D, Kim HJ, Lee C-H, Yang JI, Kim W, et al. Hepatic Steatosis Index: A Simple Screening Tool Reflecting Nonalcoholic Fatty Liver Disease. Dig Liver Dis (2010) 42:503-8. doi: 10.1016/j.dld.2009.08.002

42. Lichtinghagen R, Pietsch D, Bantel H, Manns MP, Brand K, Bahr MJ. The Enhanced Liver Fibrosis (ELF) Score: Normal Values, Influence Factors and Proposed Cut-Off Values. J Hepatol (2013) 59:236-42. doi: 10.1016/ j.jhep.2013.03.016

43. Loaeza-del-Castillo A, Paz-Pineda F, Oviedo-Cárdenas E, Sánchez-Ávila F, Vargas-Vorácková F. AST to Platelet Ratio Index (APRI) for the Noninvasive Evaluation of Liver Fibrosis: Original Article. Ann Hepatol (2008) 7:350-7. doi: 10.1016/S1665-2681(19)31836-8

44. He L, Deng L, Zhang Q, Guo J, Zhou J, Song W, et al. Diagnostic Value of CK-18, FGF-21, and Related Biomarker Panel in Nonalcoholic Fatty Liver Disease: A Systematic Review and Meta-Analysis. BioMed Res Int (2017) 2017:9729107. doi: 10.1155/2017/9729107

45. Xu Z, Zhang X, Lau J, Yu J. C-X-C Motif Chemokine 10 in Non-Alcoholic Steatohepatitis: Role as a Pro-Inflammatory Factor and Clinical Implication. Expert Rev Mol Med (2016) 18:e16. doi: 10.1017/erm.2016.16

46. Daniels SJ, Leeming DJ, Eslam M, Hashem AM, Nielsen MJ, Krag A, et al. ADAPT: An Algorithm Incorporating PRO-C3 Accurately Identifies Patients With NAFLD and Advanced Fibrosis. Hepatology (2019) 69:1075-86. doi: 10.1002/hep.30163

47. Sanyal AJ, Friedman SL, McCullough AJ, Dimick-Santos LAmerican Association for the Study of Liver Diseases, United States Food and Drug Administration. Challenges and Opportunities in Drug and Biomarker Development for Nonalcoholic Steatohepatitis: Findings and Recommendations From an American Association for the Study of Liver Diseases-U.S. Food and Drug Administration Joint Workshop. Hepatology (2015) 61:1392-405. doi: 10.1002/hep.27678

48. Kleiner DE, Brunt EM, Van Natta M, Behling C, Contos MJ, Cummings OW, et al. Design and Validation of a Histological Scoring System for Nonalcoholic Fatty Liver Disease. Hepatology (2005) 41:1313-21. doi: 10.1002/hep.20701

49. Brunt EM, Kleiner DE, Wilson LA, Belt P, Neuschwander-Tetri BANASH Clinical Research Network (CRN). Nonalcoholic Fatty Liver Disease (NAFLD) Activity Score and the Histopathologic Diagnosis in NAFLD: Distinct Clinicopathologic Meanings. Hepatology (2011) 53:810-20. doi: 10.1002/hep.24127

50. Puri P, Sanyal AJ. Nonalcoholic Fatty Liver Disease: Definitions, Risk Factors, and Workup. Clin Liver Dis (2012) 1:99-103. doi: 10.1002/cld.81

51. Brunt EM, Janney CG, Di Bisceglie AM, Neuschwander-Tetri BA, Bacon BR. Nonalcoholic Steatohepatitis: A Proposal for Grading and Staging the Histological Lesions. Am J Gastroenterol (1999) 94:2467-74. doi: 10.1111/ j.1572-0241.1999.01377.x

52. Bedossa P, Poitou C, Veyrie N, Bouillot J-L, Basdevant A, Paradis V, et al. Histopathological Algorithm and Scoring System for Evaluation of Liver Lesions in Morbidly Obese Patients. Hepatology (2012) 56:1751-9. doi: 10.1002/hep.25889

53. Ratziu V, Charlotte F, Heurtier A, Gombert S, Giral P, Bruckert E, et al. Sampling Variability of Liver Biopsy in Nonalcoholic Fatty Liver Disease. Gastroenterology (2005) 128:1898-906. doi: 10.1053/j.gastro.2005.03.084

54. Kuwashiro T, Takahashi H, Hyogo H, Ogawa Y, Imajo K, Yoneda M, et al. Discordant Pathological Diagnosis of Non-Alcoholic Fatty Liver Disease: A Prospective Multicenter Study. JGH Open (2020) 4:497-502. doi: 10.1002/ jgh3.12289

55. Juluri R, Vuppalanchi R, Olson J, Unalp A, Van Natta ML, Cummings OW, et al. Generalizability of the Nonalcoholic Steatohepatitis Clinical Research Network Histologic Scoring System for Nonalcoholic Fatty Liver Disease. J Clin Gastroenterol (2011) 45:55-8. doi: 10.1097/MCG.0b013e3181dd1348

56. Mancina RM, Dongiovanni P, Petta S, Pingitore P, Meroni M, Rametta R, et al. The MBOAT7-TMC4 Variant Rs641738 Increases Risk of Nonalcoholic Fatty Liver Disease in Individuals of European Descent. Gastroenterology (2016) 150:1219-30.e6. doi: 10.1053/j.gastro.2016.01.032

57. Zhang YN, Fowler KJ, Hamilton G, Cui JY, Sy EZ, Balanay M, et al. Liver Fat Imaging-A Clinical Overview of Ultrasound, CT, and MR Imaging. $\mathrm{Br} J$ Radiol (2018) 91:20170959. doi: 10.1259/bjr.20170959 
58. Li Q, Dhyani M, Grajo JR, Sirlin C, Samir AE. Current Status of Imaging in Nonalcoholic Fatty Liver Disease. World J Hepatol (2018) 10:530-42. doi: 10.4254/wjh.v10.i8.530

59. Loomba R. Role of Imaging-Based Biomarkers in NAFLD: Recent Advances in Clinical Application and Future Research Directions. J Hepatol (2018) 68:296-304. doi: 10.1016/j.jhep.2017.11.028

60. Bohte AE, van Werven JR, Bipat S, Stoker J. The Diagnostic Accuracy of US, CT, MRI and 1H-MRS for the Evaluation of Hepatic Steatosis Compared With Liver Biopsy: A Meta-Analysis. Eur Radiol (2011) 21:87-97. doi: 10.1007/s00330-010-1905-5

61. Di Martino M, Pacifico L, Bezzi M, Di Miscio R, Sacconi B, Chiesa C, et al. Comparison of Magnetic Resonance Spectroscopy, Proton Density Fat Fraction and Histological Analysis in the Quantification of Liver Steatosis in Children and Adolescents. World J Gastroenterol (2016) 22:8812-9. doi: 10.3748/wjg.v22.i39.8812

62. Dulai PS, Sirlin CB, Loomba R. MRI and MRE for Non-Invasive Quantitative Assessment of Hepatic Steatosis and Fibrosis in NAFLD and NASH: Clinical Trials to Clinical Practice. J Hepatol (2016) 65:1006-16. doi: 10.1016/j.jhep.2016.06.005

63. Friedman SL, Neuschwander-Tetri BA, Rinella M, Sanyal AJ. Mechanisms of NAFLD Development and Therapeutic Strategies. Nat Med (2018) 24:90822. doi: 10.1038/s41591-018-0104-9

64. Banerjee R, Pavlides M, Tunnicliffe EM, Piechnik SK, Sarania N, Philips $\mathrm{R}$, et al. Multiparametric Magnetic Resonance for the Non-Invasive Diagnosis of Liver Disease. J Hepatol (2014) 60:69-77. doi: 10.1016/j.jhep. 2013.09.002

65. Pavlides M, Banerjee R, Tunnicliffe EM, Kelly C, Collier J, Wang LM, et al. Multiparametric Magnetic Resonance Imaging for the Assessment of NonAlcoholic Fatty Liver Disease Severity. Liver Int (2017) 37:1065-73. doi: 10.1111/liv.13284

66. Dennis A, Kelly MD, Fernandes C, Mouchti S, Fallowfield JA, Hirschfield G, et al. Correlations Between MRI Biomarkers PDFF and Ct1 With Histopathological Features of Non-Alcoholic Steatohepatitis. Front Endocrinol (2020) 11:575843. doi: 10.3389/fendo.2020.575843

67. Neuschwander-Tetri BA, Clark JM, Bass NM, Van Natta ML, Unalp-Arida A, Tonascia J, et al. Clinical, Laboratory and Histological Associations in Adults With Nonalcoholic Fatty Liver Disease. Hepatology (2010) 52:91324. doi: 10.1002/hep. 23784

68. Sanyal AJAmerican Gastroenterological Association. AGA Technical Review on Nonalcoholic Fatty Liver Disease. Gastroenterology (2002) 123:1705-25. doi: 10.1053/gast.2002.36572

69. Bayard M, Holt JD, Boroughs E. Nonalcoholic Fatty Liver Disease (2006). Available at: https://www.aafp.org/afp/2006/0601/p1961.html (Accessed 19 Oct 2021).

70. Wong VW-S, Wong GL-H, Tsang SW-C, Hui AY, Chan AW-H, Choi PC-L, et al. Metabolic and Histological Features of Non-Alcoholic Fatty Liver Disease Patients With Different Serum Alanine Aminotransferase Levels. Aliment Pharmacol Ther (2009) 29:387-96. doi: 10.1111/j.1365-2036. 2008.03896.x

71. Ma X, Liu S, Zhang J, Dong M, Wang Y, Wang M, et al. Proportion of NAFLD Patients With Normal ALT Value in Overall NAFLD Patients: A Systematic Review and Meta-Analysis. BMC Gastroenterol (2020) 20:10. doi: 10.1186/s12876-020-1165-Z

72. Anstee QM, Day CP. The Genetics of NAFLD. Nat Rev Gastroenterol Hepatol (2013) 10:645-55. doi: 10.1038/nrgastro.2013.182

73. Altshuler D, Daly MJ, Lander ES. Genetic Mapping in Human Disease. Science (2008) 322:881-8. doi: 10.1126/science.1156409

74. Hirschhorn JN. Genomewide Association Studies-Illuminating Biologic Pathways. N Engl J Med (2009) 360:1699-701. doi: 10.1056/ NEJMp0808934

75. Manolio TA, Collins FS, Cox NJ, Goldstein DB, Hindorff LA, Hunter DJ, et al. Finding the Missing Heritability of Complex Diseases. Nature (2009) 461:747-53. doi: 10.1038/nature08494

76. Liu DJ, Peloso GM, Yu H, Butterworth AS, Wang X, Mahajan A, et al. Exome-Wide Association Study of Plasma Lipids in $>300,000$ Individuals. Nat Genet (2017) 49:1758-66. doi: 10.1038/ng.3977

77. Rabbani B, Tekin M, Mahdieh N. The Promise of Whole-Exome Sequencing in Medical Genetics. J Hum Genet (2014) 59:5-15. doi: 10.1038/jhg.2013.114
78. Eslam M, Valenti L, Romeo S. Genetics and Epigenetics of NAFLD and NASH: Clinical Impact. J Hepatol (2018) 68:268-79. doi: 10.1016/j.jhep. 2017.09.003

79. Trépo E, Valenti L. Update on NAFLD Genetics: From New Variants to the Clinic. J Hepatol (2020) 72:1196-209. doi: 10.1016/j.jhep.2020.02.020

80. Abul-Husn NS, Cheng X, Li AH, Xin Y, Schurmann C, Stevis P, et al. A Protein-Truncating HSD17B13 Variant and Protection From Chronic Liver Disease. N Engl J Med (2018) 378:1096-106. doi: 10.1056/NEJMoa1712191

81. Namjou B, Lingren T, Huang Y, Parameswaran S, Cobb BL, Stanaway IB, et al. GWAS and Enrichment Analyses of Non-Alcoholic Fatty Liver Disease Identify New Trait-Associated Genes and Pathways Across Emerge Network. BMC Med (2019) 17:135. doi: 10.1186/s12916-019-1364-z

82. Anstee QM, Darlay R, Cockell S, Meroni M, Govaere O, Tiniakos D, et al. Genome-Wide Association Study of non-Alcoholic Fatty Liver and Steatohepatitis in a Histologically Characterised Cohort tr. J Hepatol (2020) 73:505-15. doi: 10.1016/j.jhep.2020.04.003

83. Emdin CA, Haas ME, Khera AV, Aragam K, Chaffin M, Klarin D, et al. A Missense Variant in Mitochondrial Amidoxime Reducing Component 1 Gene and Protection Against Liver Disease. PloS Genet (2020) 16:e1008629. doi: 10.1371/journal.pgen.1008629

84. Parisinos CA, Wilman HR, Thomas EL, Kelly M, Nicholls RC, McGonigle J, et al. Genome-Wide and Mendelian Randomisation Studies of Liver MRI Yield Insights Into the Pathogenesis of Steatohepatitis. J Hepatol (2020) 73:241-51. doi: 10.1016/j.jhep.2020.03.032

85. Jamialahmadi O, Mancina RM, Ciociola E, Tavaglione F, Luukkonen PK, Baselli G, et al. Exome-Wide Association Study on Alanine Aminotransferase Identifies Sequence Variants in the GPAM and APOE Associated With Fatty Liver Disease. Gastroenterology (2021) 160:1634-46.e7. doi: 10.1053/ j.gastro.2020.12.023

86. Palmer ND, Kahali B, Kuppa A, Chen Y, Du X, Feitosa MF, et al. AlleleSpecific Variation at APOE Increases Nonalcoholic Fatty Liver Disease and Obesity But Decreases Risk of Alzheimer's Disease and Myocardial Infarction. Hum Mol Genet (2021) 30:1443-56. doi: 10.1093/hmg/ddab096

87. Pazoki R, Vujkovic M, Elliott J, Evangelou E, Gill D, Ghanbari M, et al. Genetic Analysis in European Ancestry Individuals Identifies 517 Loci Associated With Liver Enzymes. Nat Commun (2021) 12:1-12. doi: 10.1038/s41467-021-22338-2

88. Liu Y, Basty N, Whitcher B, Bell JD, Sorokin EP, van Bruggen N, et al. Genetic Architecture of 11 Organ Traits Derived From Abdominal MRI Using Deep Learning. Elife (2021) 10:e65554. doi: 10.7554/eLife.65554

89. Liu Y-L, Patman GL, Leathart JBS, Piguet A-C, Burt AD, Dufour J-F, et al. Carriage of the PNPLA3 Rs738409 C >G Polymorphism Confers an Increased Risk of Non-Alcoholic Fatty Liver Disease Associated Hepatocellular Carcinoma. J Hepatol (2014) 61:75-81. doi: 10.1016/j.jhep. 2014.02.030

90. Kozlitina J, Smagris E, Stender S, Nordestgaard BG, Zhou HH, TybjærgHansen A, et al. Exome-Wide Association Study Identifies a TM6SF2 Variant That Confers Susceptibility to Nonalcoholic Fatty Liver Disease. Nat Genet (2014) 46:352-6. doi: 10.1038/ng.2901

91. Liu Y-L, Reeves HL, Burt AD, Tiniakos D, McPherson S, Leathart JBS, et al. TM6SF2 Rs58542926 Influences Hepatic Fibrosis Progression in Patients With Non-Alcoholic Fatty Liver Disease. Nat Commun (2014) 5:4309. doi: 10.1038/ncomms5309

92. Chambers JC, Zhang W, Sehmi J, Li X, Wass MN, van der Harst P, et al. Genome-Wide Association Study Identifies Loci Influencing Concentrations of Liver Enzymes in Plasma. Nat Genet (2011) 43:1131-8. doi: 10.1038/ ng. 970

93. Palmer ND, Musani SK, Yerges-Armstrong LM, Feitosa MF, Bielak LF, Hernaez R, et al. Characterization of European Ancestry Nonalcoholic Fatty Liver Disease-Associated Variants in Individuals of African and Hispanic Descent. Hepatology (2013) 58:966-75. doi: 10.1002/hep.26440

94. Speliotes EK, Yerges-Armstrong LM, Wu J, Hernaez R, Kim LJ, Palmer CD, et al. Genome-Wide Association Analysis Identifies Variants Associated With Nonalcoholic Fatty Liver Disease That Have Distinct Effects on Metabolic Traits. PloS Genet (2011) 7:e1001324. doi: 10.1371/journal. pgen. 1001324

95. Buch S, Stickel F, Trépo E, Way M, Herrmann A, Nischalke HD, et al. A Genome-Wide Association Study Confirms PNPLA3 and Identifies TM6SF2 
and MBOAT7 as Risk Loci for Alcohol-Related Cirrhosis. Nat Genet (2015) 47:1443-8. doi: 10.1038/ng.3417

96. Alam S, Islam MS, Islam S, Mustafa G, Saleh AA, Ahmad N. Association of Single Nucleotide Polymorphism at PNPLA3 With Fatty Liver, Steatohepatitis, and Cirrhosis of Liver. Indian J Gastroenterol (2017) 36:366-72. doi: 10.1007/s12664-017-0784-y

97. Romeo S, Kozlitina J, Xing C, Pertsemlidis A, Cox D, Pennacchio LA, et al. Genetic Variation in PNPLA3 Confers Susceptibility to Nonalcoholic Fatty Liver Disease. Nat Genet (2008) 40:1461-5. doi: 10.1038/ng.257

98. Teo K, Abeysekera KWM, Adams L, Aigner E, Anstee QM, Banales JM, et al. Rs641738c $>$ T Near MBOAT7 Is Associated With Liver Fat, ALT and Fibrosis in NAFLD: A Meta-Analysis. J Hepatol (2021) 74:20-30. doi: 10.1016/j.jhep.2020.08.027

99. Tam V, Patel N, Turcotte M, Bossé Y, Paré G, Meyre D. Benefits and Limitations of Genome-Wide Association Studies. Nat Rev Genet (2019) 20:467-84. doi: 10.1038/s41576-019-0127-1

100. Long AD, Langley $\mathrm{CH}$. The Power of Association Studies to Detect the Contribution of Candidate Genetic Loci to Variation in Complex Traits. Genome Res (1999) 9:720-31. doi: 10.1101/gr.9.8.720

101. Kathiresan S, Melander O, Guiducci C, Surti A, Burtt NP, Rieder MJ, et al. Six New Loci Associated With Blood Low-Density Lipoprotein Cholesterol, High-Density Lipoprotein Cholesterol or Triglycerides in Humans. Nat Genet (2008) 40:189-97. doi: 10.1038/ng.75

102. Blanchard V, Khantalin I, Ramin-Mangata S, Chémello K, Nativel B, Lambert G. PCSK9: From Biology to Clinical Applications. Pathology (2019) 51:177-83. doi: 10.1016/j.pathol.2018.10.012

103. Kleinstein SE, Rein M, Abdelmalek MF, Guy CD, Goldstein DB, Mae Diehl A, et al. Whole-Exome Sequencing Study of Extreme Phenotypes of NAFLD. Hepatol Commun (2018) 2:1021-9. doi: 10.1002/hep4.1227

104. Haas ME, Pirruccello JP, Friedman SN, Emdin CA, Ajmera VH, Simon TG, et al. Machine Learning Enables New Insights Into Clinical Significance of and Genetic Contributions to Liver Fat Accumulation. medRxiv (2020) 2020.09.03.20187195. doi: 10.1101/2020.09.03.20187195

105. Vujkovic M, Ramdas S, Lorenz KM, Guo X, Darlay R, Cordell HJ, et al. A Trans-Ancestry Genome-Wide Association Study of Unexplained Chronic ALT Elevation as a Proxy for Nonalcoholic Fatty Liver Disease With Histological and Radiological Validation. medRxiv (2021) 2020. 12.26.20248491. doi: 10.1101/2020.12.26.20248491

106. A Study of ALN-HSD in Healthy Adult Subjects and Adult Patients With Nonalcoholic Steatohepatitis (NASH). Available at: https://clinicaltrials.gov/ ct2/show/NCT04565717 (Accessed 9 Sep 2021).

107. Gane E, Schwabe C, Yoon KT, Heo J, Scott R, Lee J-H, et al. ARO-HSD Reduces Hepatic HSD17B13 Mrna Expression and Protein Levels in Patients With Suspected NASH. Available at: https://ir.arrowheadpharma.com/staticfiles/b6b66255-5618-4706-96f2-e0fbfc68ce75.

108. Pelusi S, Baselli G, Pietrelli A, Dongiovanni P, Donati B, McCain MV, et al. Rare Pathogenic Variants Predispose to Hepatocellular Carcinoma in
Nonalcoholic Fatty Liver Disease. Sci Rep (2019) 9:3682. doi: 10.1038/ s41598-019-39998-2

109. Hakim A, Zhang X, DeLisle A, Oral EA, Dykas D, Drzewiecki K, et al. Clinical Utility of Genomic Analysis in Adults With Idiopathic Liver Disease. J Hepatol (2019) 70:1214-21. doi: 10.1016/j.jhep.2019.01.036

110. Eslam M, Sanyal AJ, George JInternational Consensus Panel. MAFLD: A Consensus-Driven Proposed Nomenclature for Metabolic Associated Fatty Liver Disease. Gastroenterology (2020) 158:1999-2014.e1. doi: 10.1053/ j.gastro.2019.11.312

111. Eslam M, Newsome PN, Sarin SK, Anstee QM, Targher G, Romero-Gomez M, et al. A New Definition for Metabolic Dysfunction-Associated Fatty Liver Disease: An International Expert Consensus Statement. J Hepatol (2020) 73:202-9. doi: 10.1016/j.jhep.2020.07.045

112. Lin S, Huang J, Wang M, Kumar R, Liu Y, Liu S, et al. Comparison of MAFLD and NAFLD Diagnostic Criteria in Real World. Liver Int (2020) 40:2082-9. doi: 10.1111/liv.14548

113. Yamamura S, Eslam M, Kawaguchi T, Tsutsumi T, Nakano D, Yoshinaga S, et al. MAFLD Identifies Patients With Significant Hepatic Fibrosis Better Than NAFLD. Liver Int (2020) 40:3018-30. doi: 10.1111/liv.14675

114. Niriella MA, Ediriweera DS, Kasturiratne A, De Silva ST, Dassanayaka AS, De Silva AP, et al. Outcomes of NAFLD and MAFLD: Results From a Community-Based, Prospective Cohort Study. PloS One (2021) 16:e245762. doi: 10.1371/journal.pone.0245762

115. Younossi ZM, Rinella ME, Sanyal AJ, Harrison SA, Brunt EM, Goodman Z, et al. From NAFLD to MAFLD: Implications of a Premature Change in Terminology. Hepatology (2021) 73:1194-8. doi: 10.1002/hep.31420

116. Xia M, Zeng H, Wang S, Tang H, Gao X. Insights Into Contribution of Genetic Variants Towards the Susceptibility of MAFLD Revealed by the NMR-Based Lipoprotein Profiling. J Hepatol (2021) 74:974-7. doi: 10.1016/ j.jhep.2020.10.019

Conflict of Interest: The authors declare that the research was conducted in the absence of any commercial or financial relationships that could be construed as a potential conflict of interest.

Publisher's Note: All claims expressed in this article are solely those of the authors and do not necessarily represent those of their affiliated organizations, or those of the publisher, the editors and the reviewers. Any product that may be evaluated in this article, or claim that may be made by its manufacturer, is not guaranteed or endorsed by the publisher.

Copyright (C) $2021 \mathrm{Du}$, DeForest and Majithia. This is an open-access article distributed under the terms of the Creative Commons Attribution License (CC BY). The use, distribution or reproduction in other forums is permitted, provided the original author(s) and the copyright owner(s) are credited and that the original publication in this journal is cited, in accordance with accepted academic practice. No use, distribution or reproduction is permitted which does not comply with these terms. 